\title{
Therapeutic potential of bone marrow-derived mesenchymal stem cells producing pigment epithelium-derived factor in lung carcinoma
}

\author{
QIAOLING CHEN, PING CHENG, TAO YIN, HONG HE, LI YANG, YUQUAN WEI and XIANCHENG CHEN \\ State Key Laboratory of Biotherapy and Cancer Center, West China Hospital, West China \\ Medical School, Sichuan University, Chengdu, Sichuan, P.R. China
}

Received February 13, 2012; Accepted April 6, 2012

DOI: 10.3892/ijmm.2012.1015

\begin{abstract}
Specific and efficient gene delivery to target cells and the subsequent expression of the RNA and protein is crucial to the success of gene-based therapy for cancer. Mesenchymal stem cells (MSCs) represent novel and efficient tools for delivery of therapeutic agents to tumor cells. In this study, we evaluated the potential of bone marrow-derived mesenchymal stem cells, genetically modified to express pigment epithelium-derived factor (PEDF) for the treatment of Lewis lung carcinoma (LLC). MSCs derived from murine bone marrow were efficiently engineered to express human PEDF by adenoviral transduction, and the expression and bioactivity of the transgenic protein from engineered MSCs were confirmed in vitro. Animal experiments showed that the systemic administration of MSCs treated with PEDF dramatically reduced the growth of LLC tumors and significantly prolonged survival. Immunohistochemistry analysis of the tumors from MSC-PEDF-treated animals indicated an increase in apoptosis and a decrease in microvessel density. ELISA showed that the group of MSCs treated with PEDF had relatively higher expression levels of PEDF in tumor tissue and lower levels in serum compared with the free Ad-PEDF group. These results suggest that MSCs have potential use as effective delivery of vehicles for therapeutic genes in the treatment of LLC.
\end{abstract}

\section{Introduction}

Lung cancer is one of the most common malignant tumors in humans and has high morbidity and mortality. Worldwide,

Correspondence to: Dr Xiancheng Chen, State Key Laboratory of Biotherapy and Cancer Center, West China Hospital, Sichuan University, 1 Keyuan Road 4, Gaopeng Street, High Technological Development Zone, Chengdu 610041, P.R. China

E-mail: chenwcrs@126.com; blww25@163.com

Key words: mesenchymal stem cells, pigment epithelium-derived factor, lung carcinoma, gene therapy, anti-angiogenesis over 1.3 million people are diagnosed each year with lung cancer, with over 1.1 million deaths $(1,2)$. Non-small cell lung cancer (NSCLC) is the most common type of lung cancer, accounting for $85 \%$ of cases. The prognosis of patients with NSCLC is extremely poor because most patients are diagnosed in an advanced stage with local or distant metastases. Surgical treatment is reserved only for patients with early stage disease. Radiotherapy and chemotherapy are not specific to cancer cells and produce severe side effects, including bone marrow suppression, renal impairment and second cancer occurrence $(3,4)$. Therefore, development of new therapeutic strategies is an urgent need for the treatment of NSCLC.

Angiogenesis, the outgrowth of new blood vessels from pre-existing ones, plays an essential role in tumor growth and metastasis (5). A positive association between tumor angiogenesis and tumor aggressiveness in carcinoma of the breast, lung, prostate, malignant melanoma and other solid tumors has been confirmed by many clinical studies (6). Thus, depriving a tumor of its vascular supply with anti-angiogenic agents is a promising approach against tumors (7). Pigment epithelium-derived factor (PEDF), a $50 \mathrm{kDa}$ secreted glycoprotein that belongs to the non-inhibitory serpin group, is a potent endogenous inhibitor of angiogenesis $(8,9)$. PEDF exerts its antitumor effects via antiangiogenesis, tumor differentiation, and direct tumor suppression by apoptosis (8). Much focus on PEDF as a promising therapeutic target in cancer has stemmed from its potent antiangiogenic activity, which has shown to be more effective than any other known endogenous angiogenic inhibitor, including angiostatin, thrombospondin-1, and endostatin (10). It has been demonstrated that PEDF can inhibit the proliferation and migration of endothelial cells and can induce apoptosis (8). A number of studies showed inverse correlation between PEDF levels, grade and metastatic potential of prostate cancer (11), pancreatic cancer (12), glioma (13), cervical carcinoma (14) as well as lung carcinoma (15).

Marrow-derived stromal cells (MSCs), also known as mesenchymal stem cells or nonhematopoietic progenitor cells, are precursors that have the potential to differentiate along osteogenic, adipogenic and chondrogenic lineages when placed in the appropriate environments. This differentiation plasticity makes MSCs the ultimate candidates for future utilization 
in cell therapy and tissue regeneration $(16,17)$. Furthermore, MSCs expressing transgenes maintained long-term expression (up to 6 months) in vivo because of their hypoimmunogenic properties and production of immunosuppressive molecules $(18,19)$. The tumor microenvironment is known to provide a preferential niche for MSCs homing and survival. As MSCs have the capacity to home to the tumor microenvironment, they may be promising tools for the selective delivery of antineoplastic drugs to tumors $(20,21)$.

In this study, we developed a novel strategy of tumor gene therapy in which MSCs loaded with recombinant adenoviruses expressing PEDF could effectively suppress tumor growth and prolong the lifespan through inhibiting angiogenesis in a mouse model, indicating that MSCs may be employed as an effective carrier for tumor gene therapy.

\section{Materials and methods}

Cell lines and culture. Lewis lung carcinoma (LL/2) and human embryonic kidney 293 cell lines (purchased from American Type Culture Collection, ATCC, Rockville, MD) were maintained in Dulbecco's modified Eagle's medium (Gibco-BRL, Grand Island, NY) supplemented with $10 \%$ heat-inactivated fetal bovine serum (Gibco, Auckland, NZ), $2 \mathrm{mM} \mathrm{L-glutamine}$ and $100 \mu \mathrm{g} / \mathrm{ml}$ of Amikacin. Human umbilical vein endothelial cells (HUVECs) were isolated from human umbilical cord veins as previously described (22), and grown in EBM-2 medium with SingleQuots (Lonza) containing VEGF and other growth factors. HUVECs at passages 2 to 6 were used for all experiments.

Isolation and culture of marrow MSCs. Female C57BL/6 mice 4- to 6-week-old were sacrificed, femurs and tibias were isolated, and whole bone marrow was retrieved by flushing these bones with low-glucose DMEM (Gibco), supplemented with $10 \% \mathrm{FBS}$ and $50 \mathrm{U} / \mathrm{ml}$ penicillin, $50 \mu \mathrm{g} / \mathrm{ml}$ streptomycin. All of these bone marrow cells were then cultured at $37^{\circ} \mathrm{C}$ in a $5 \% \mathrm{CO}_{2}$ and a $95 \%$ humidified atmosphere. Two days later, the nonadherent hematopoietic cells were discarded and the adherent MSCs were preserved for further expansion. The medium was changed twice/week and the MSCs were cultured for 14-days before first passage. Cells of 4-5 passages were used in the experiments (23). MSCs have been proved to express several cell lineage-specific antigens (24). Therefore, the plate-adhering cells were further sorted by flow cytometry using CD34, CD44, CD45, CD73, CD90 and CD105 (BD Biosciences) for phenotype characteristics of MSCs. Isotypic control analyses were conducted in parallel. Flow cytometric analysis was performed on a BD Biosciences FACSCalibur flow cytometer, and data were analyzed with the CellQuest Pro software (BD Immunocytometry Systems).

Adenoviral transduction of MSCs. The adenoviruses were created using the AdEasy system. The viruses were amplified in $\mathrm{HEK} 293$ cells and purified on $\mathrm{CsCl}$ gradients according to standard methods (25). When the confluence of proliferating MSCs reached or surpassed $\sim 90 \%$, the cells were transduced with recombinant adenovirus at a multiplicity of infection (MOI) of 1,500 for $4 \mathrm{~h}$. MSCs were also infected with adenovirus-LacZ (Ad-LacZ) at an MOI of 1,500 as a control.
Verification of expressed PEDF from MSCs-PEDF in vitro. Western blot analysis was performed as described previously (26). Briefly, MSCs were infected with adenoviruses for $4 \mathrm{~h}$ and then the virus-containing medium was removed and additionally incubated in low-serum medium (low-glucose DMEM containing $2 \% \mathrm{FBS}$ ). After a further $48 \mathrm{~h}$ of incubation, the conditioned media was collected. The secreted proteins in the supernatants of the culture were precipitated by TCA-DOC/ acetone and the western blotting assay was performed using a standard method with a mouse anti-human PEDF monoclonal antibody (R\&D Systems, Boston, MA, USA) and a biotinylated secondary antibody, and the bands were detected using an enhanced chemiluminescence detection system (Pierce, Rockford, IL, USA). The concentration of the PEDF secreted in the culture supernatants was detected using a sandwich enzyme-linked immunosorbent assay (ELISA) kit for the human PEDF protein (Groundwork Biotechnology Diagnosticate Ltd., San Diego, CA, USA) following the manufacturer's protocol.

Tube formation assay. The tube formation assay was conducted as described previously (27) to evaluate the antiangiogenic activity of PEDF produced by MSCs-PEDF. Each well of a 96-well plate was coated with $50 \mu 1$ Matrigel (BD Biosciences). After polymerization at $37^{\circ} \mathrm{C}$ for $30 \mathrm{~min}$, HUVECs $\left(2 \times 10^{4} /\right.$ well) were suspended in $200 \mu 1$ of the conditioned media derived from MSCs, MSCs-LacZ, MSCs-PEDF respectively and seeded onto the Matrigel. Six hours later, cells were viewed and photographed under an inverted microscope with a digital camera. The tubes of five fields were counted and the data were averaged.

Transwell invasion assay in vitro. To determine whether PEDF secreted by MSCs-PEDF can inhibit HUVEC invasion, a Transwell invasion assay was performed as previously described (28). Briefly, the filter of the Transwell chamber (Millipore) was coated with $50 \mu 1$ Matrigel (BD Biosciences). After Matrigel polymerization, HUVEC (2x10/well) were suspended in $200 \mu 1$ of the conditioned media derived from MSCs, MSCs-LacZ, MSCs-PEDF respectively and loaded in the upper chamber. The lower well of the Transwell plate was filled with $600 \mu 1$ of EBM-2 medium containing various growth factors. Cells were allowed to migrate for $24 \mathrm{~h}$ at $37^{\circ} \mathrm{C}$. Non-migrated cells were scraped with a cotton swab, and migrated cells were fixed with $100 \%$ methanol and stained with $0.05 \%$ crystal violet. The number of cells that had migrated to the lower side of the filter was quantified by manual counting under a light microscope with five fields (x100). All assays were conducted in triplicate.

Alginate encapsulation assay. An alginate-encapsulated tumor cell assay was conducted as previously described (26). Alginate beads were formed with about $1 \times 10^{5}$ LLC cells per bead and implanted s.c. into both dorsal sides of C57BL/6 mice. Then the mice were injected with PBS, MSCs-LacZ, MSCsPEDF or free Ad-PEDF via the tail vein on Day 2 for once. Twelve days after the beads were implanted, the mice were injected intravenously with a dose at $100 \mathrm{mg} / \mathrm{kg}$ FITC-dextran solution (Sigma) in $100 \mu$ l. Alginate beads were removed and photographed with a digital camera within 20 min after FITC- 
dextran administration. The uptake of FITC-dextran was quantified against a calibration curve of FITC-dextran.

PEDF-expressing MSCs reduce subcutaneous tumor growth in vivo. Female syngeneic C57BL/6 mice 6-8 weeks of age were purchased from the West China Experimental Animal Center of Sichuan University (Sichuan, China) and maintained in a dedicated pathogen-free environment. All animal procedures were approved by the Institute's Animal Care and Use Committee. Mice were implanted s.c. in the right flank with LLC cells at $5 \times 10^{5} /$ mouse. When tumor diameters reached $3 \mathrm{~mm}$, mice were randomly divided into four groups and i.v. injected with $\mathrm{PBS}, 10^{8}$ plaque forming units (PFU) of Ad-PEDF, $5 \times 10^{5}$ MSCs-LacZ or $5 \times 10^{5}$ MSCs-PEDF at 4 days intervals for two times. Tumor growth and survival rate were monitored every 3 days by caliper. Tumor volumes were determined at various time points using the formula: width ${ }^{2}$ $x$ length $x$ 0.52. Cohorts of mice $(n=8)$ from each group were sacrificed at Day-30 to determine the effect of therapy and remaining mice $(n=10)$ were monitored for long-term survival. The duration of survival was recorded when the mouse died or had to be sacrificed secondary to tumor diameter $>20 \mathrm{~mm}$, tumor ulceration or bleeding. The difference in survival was determined by a log-rank test.

Immunofluorescence staining of tumor vessels. To determine the degree of tumor-induced angiogenesis, frozen sections were prepared from the tumor tissue 30 days after inoculation. The procedure of immunofluorescence staining was done as described previously (29). Briefly, sections were probed with a monoclonal anti-CD31 antibody (BD Biosciences) and nuclei were stained with DAPI. The sections were viewed at low magnification $(\mathrm{x} 40)$ to identify the most vascular-rich area (hot spot) and 5 non-overlapping fields were selected. Microvessel counting was performed at a high-power field (x200) under fluorescence microscopy and data were expressed as the mean number of CD31-positive vessels per field from three sections in each tumor.

TUNEL assay for in situ apoptosis. To determine the degree of apoptosis, terminal dUTP nick-end labeling (TUNEL) staining was performed using an in situ cell death detection kit (Promega) following the manufacturer's protocol. Tumor species were prepared as described above. Three tumors from each group were analyzed. The number of apoptotic cells was quantified in 5 randomly selected fields at x200 magnifications using a fluorescence microscope.

ELISA for expressed PEDF in serum and tumor tissue. Serum samples and fresh tumor tissues were harvested and detected using ELISA (Groundwork Biotechnology Diagnosticate, Ltd.) in triplicate for the tendencies of PEDF levels at 7, 14 and 21 days after i.v. administration of treatment. Non-necrotic fresh tumor tissues were cut into small pieces, lysed in RIPA buffer on ice, passed through a sieve (BellCo Glass), and evaluated for the local production of PEDF as $\mathrm{ng} / \mathrm{mg}$.

Statistical analysis. All values are presented as means \pm SEM (standard error of the mean). SPSS 17.0 was used for statistical analysis. Significance was evaluated using one-way ANOVA, and $\mathrm{P}<0.05$ was considered significant differences. The survival rates were compared by means of the log-rank test.

\section{Results}

MSCs isolation, genetic modification and confirmation of PEDF expression in vitro. The adherent cells have a typical spindle-like morphology (Fig. 1B). Analysis of immunophenotype showed that isolated BM-derived cells shared classical immunophenotype of MSCs, including positivity for CD44, CD73, CD90, CD105, but negativity for CD34, CD45 (Fig. 1A). Thus, as the data showed, we successfully obtained abundant MSCs from mouse bone marrow.

After BMSCs were cultured to reach $90 \%$ confluence and incubated with adenoviruses at a MOI of 1,500 for $4 \mathrm{~h}$, the secreted PEDF in the supernatants of conditioned media was verified by western blotting. As shown in Fig. 1C, a specific $50 \mathrm{kDa}$ band was observed only in the supernatants from Ad-PEDF-transduced MSCs, which indicated that PEDF was appropriately synthesized and processed. PEDF levels in the collected media $(n=3)$ were determined utilizing a sandwich enzyme-linked immunosorbent assay (ELISA) kit for the human PEDF protein (Groundwork Biotechnology Diagnosticate Ltd.). It showed that PEDF was produced in vitro in great amounts for $48 \mathrm{~h}$, with a concentration as high as $78.8 \pm 4.8 \mathrm{ng} / \mathrm{ml}$ (Fig. 1D).

Bioactivity of PEDF produced by PEDF-transduced MSCs in vitro. HUVECs when seeded on Matrigel become elongated and form capillary-like structures mimicking the in vivo neoangiogenesis process (30). We used this assay to examine the antiangiogenic activity of PEDF expressed by MSCs-PEDF. As shown in Fig. 2A, HUVECs plated on the surface of Matrigel formed capillary-like structures in the MSCs and MSCs-LacZ groups after $6 \mathrm{~h}$, however, treatment with the conditioned media (CM) from MSCs-PEDF dramatically blocked the tube formation $(\mathrm{P}<0.01)$. We also conducted Transwell invasion assays to evaluate the ability of HUVECs to pass through the Matrigel and membrane barrier of the Transwell in the presence of the CM derived from MSCs, MSCs-LacZ and MSCs-PEDF. Data showed that the CM from the MSCs-PEDF significantly inhibited the invasion properties of endothelial cells (Fig. 2B) $(\mathrm{P}<0.01)$.

Therapeutic effect of MSCs-PEDF on LLC cell growth in vivo. To learn if the PEDF secreted in vivo by PEDF gene-modified MSCs can engender a beneficial effect against LLC cells in vivo, we implanted isogenic C57BL/6 mice with LLC cells s.c. and treated with PBS, $10^{8}$ PFU of Ad-PEDF, $5 \times 10^{5}$ MSCsLacZ or $5 \times 10^{5}$ MSCs-PEDF at 4 days intervals for two times i.v. Cohorts of mice $(n=8)$ from each group were sacrificed at Day-30 to determine the effect of therapy and remaining mice $(n=10)$ were monitored for long-term survival. As shown in Fig. 3A, the tumor volume in the MSCs-PEDF group was dramatically smaller compared with that of the PBS group $\left(1038.8 \pm 139.0\right.$ vs. $2897.7 \pm 274.9 \mathrm{~mm}^{3}$, ANOVA; $\left.\mathrm{P}<0.01\right)$. By contrast, the growth rate of tumors was not affected by administration of free Ad-PEDF $\left(2622.8 \pm 359.1 \mathrm{~mm}^{3}\right)$ and MSCs transduced with Ad-LacZ $\left(3019.2 \pm 360.9 \mathrm{~mm}^{3}\right)$. These data suggest that intravenous administration of MSC-PEDF cells inhibits the growth of tumors, whereas systemically injected free Ad-PEDF or MSC-LacZ cells does not. 
A
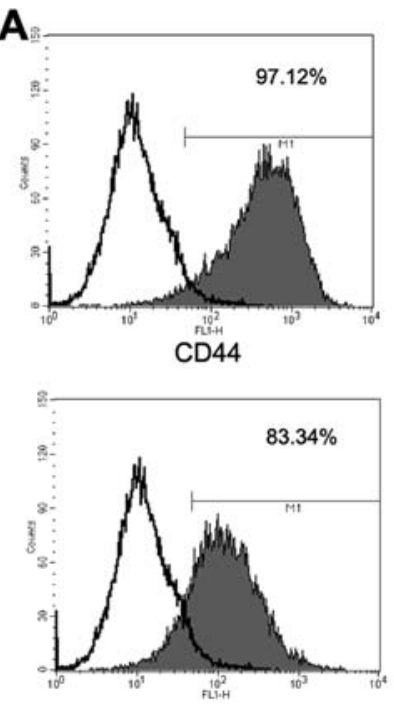

CD105

B

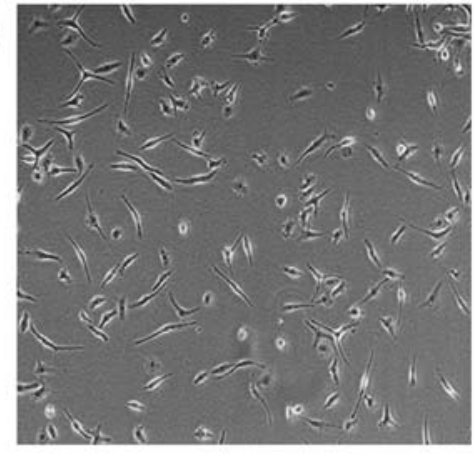

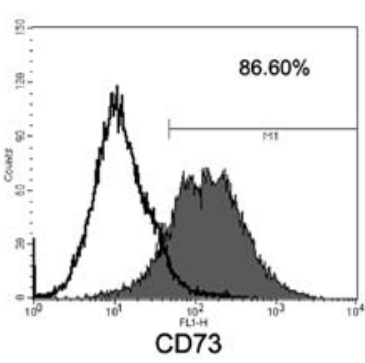
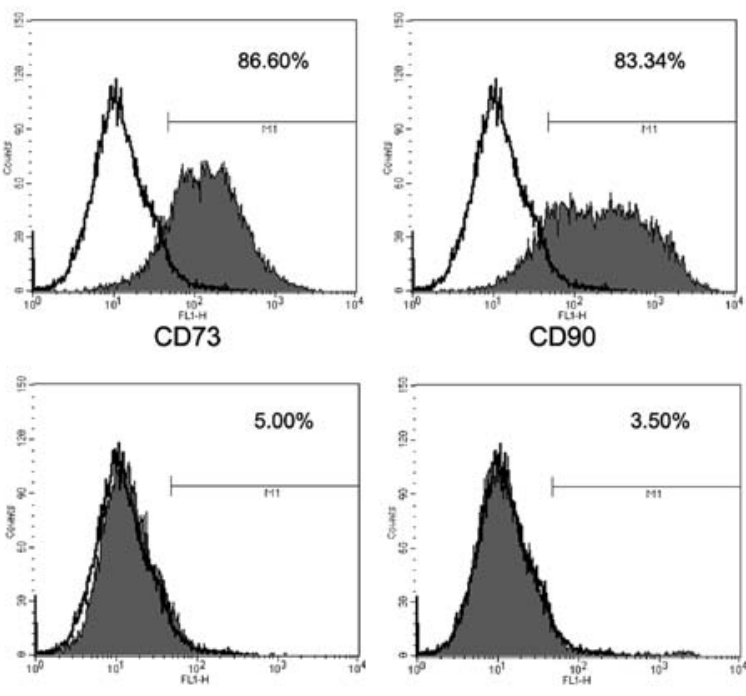

CD34

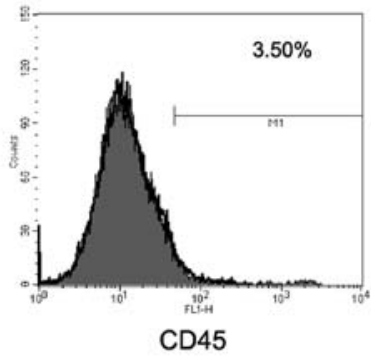

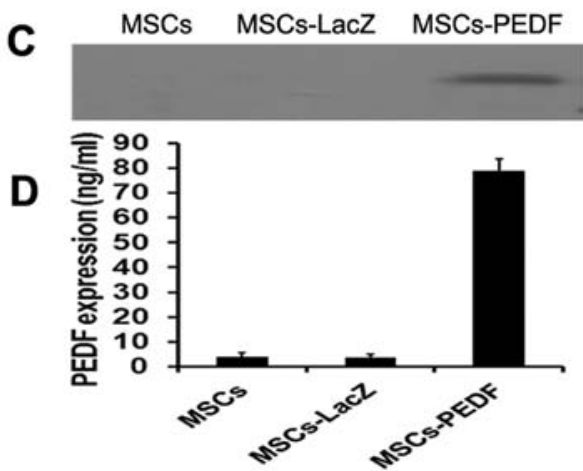

Figure 1. Characterization mouse MSCs analyzed by flow cytometry and verification of secreted PEDF by MSCs-PEDF in vitro. (A) The MSC-specific markers CD44, CD73, CD90 and CD105 were expressed in MSCs, whereas the hematopoietic stem-cell markers CD34 and CD45 were not. (B) MSCs showed a fibroblast-like morphology in vitro. (C) The conditioned media (CM) deposits from Ad-PEDF-infected MSCs could be detected by antibodies reactive to human PEDF; negative staining in supernatants deposits from control MSCs and MSC-LacZ by western blot analysis. (D) PEDF concentrations in the CM derived from untransduced hMSCs, MSCs-LacZ and MSCs-PEDF were measured by ELISA.

Moreover, MSCs producing PEDF was found to significantly increase the survival of mice. All animals in the control groups died by Day 42 , whereas $40 \%$ of mice treated with MSC-PEDF survived beyond that time $(\mathrm{P}<0.01)$. There was no significant increase in survival of mice treated with MSC transduced with Ad-LacZ or free Ad-PEDF (Fig. 3B).

Inhibition of angiogenesis in vivo. Immunofluorescence antiCD31 staining was performed to evaluate the consequence of anti-angiogenesis therapy. The most highly vascularized area of each tumor section was identified on low power and five high-powered fields were counted in this area of greatest vessel density. The tumor tissue from MSCs-PEDF-treated mice showed apparently decreased microvessel density compared with the other three control groups (Fig. 4A). The inhibition of angiogenesis was confirmed in the alginateencapsulated tumor cell assay. The treated tumor exhibited relatively little vascularity (Fig. 4B). Vascularization of beads over 12 days can then be measured by uptake of FITC-dextran into beads. Vascularization of alginate beads was apparently reduced, and FITC-dextran uptake was decreased in MSCsPEDF-treated mice compared with that in controls (ANOVA; $\mathrm{P}<0.01)$.
Induction of apoptosis in vivo. The TUNEL assay was performed to detect tumor cell apoptosis to further investigate the role of MSCs-PEDF treatment in tumors in vivo. As shown in Fig. 5, MSCs-PEDF-treated tumors showed significantly more apoptotic cells (with green nuclei) than tumors from the PBS, Ad-PEDF or MSCs-LacZ-treated groups. The apoptosis index was also significantly higher in the MSCs-PEDF-treated group compared with the controls (ANOVA; $\mathrm{P}<0.01$ ). The inhibition of angiogenesis and the consequent induction of apoptosis underlie the mechanism of tumor volume decrease and the lifespan prolongation in tumor-bearing mice.

ELISA for expressed PEDF in serum and tumor tissue. The systemic and local (serum and intratumoral) expression tendencies of PEDF at indicated points by ELISA are shown in Fig. 6. In the MSCs-PEDF group, the serum levels of PEDF were $\sim 38 \mathrm{ng} / \mathrm{ml}$ on Day 7 , and declined to $\sim 26 \mathrm{ng} / \mathrm{ml}$ after that; meanwhile, the intratumoral levels of PEDF rose from $\sim 168 \mathrm{ng} / \mathrm{mg}$ ( 7 days) to $\sim 253 \mathrm{ng} / \mathrm{mg}$ (14 days), and then slightly declined to $\sim 238 \mathrm{ng} / \mathrm{mg}$ in the 21 days, $\sim 7$-fold vs. the intratumoral level in the free Ad-PEDF group ( $32 \mathrm{ng} / \mathrm{mg}$ ). In free Ad-PEDF group, the levels of PEDF, diminished quickly from $\sim 60$ to $25 \mathrm{ng} / \mathrm{ml}$ in serum and from $\sim 50$ to $\sim 32 \mathrm{ng} / \mathrm{mg}$ 
A

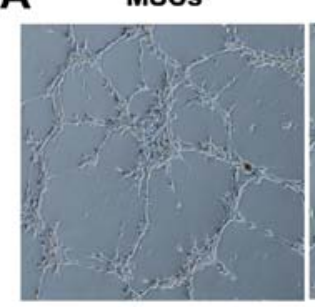

B
MSCs-LacZ

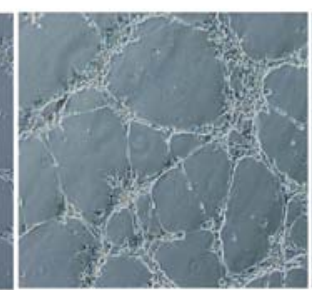

MSCs-LacZ
MSCs-PEDF

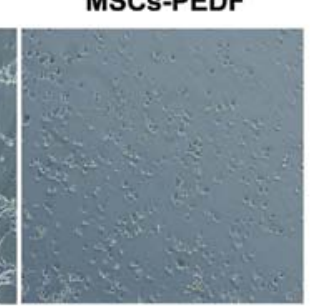

MSCS-PEDF

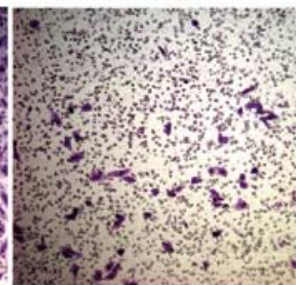

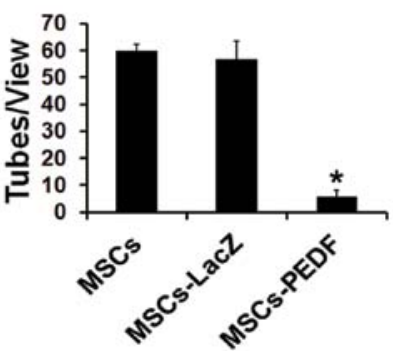

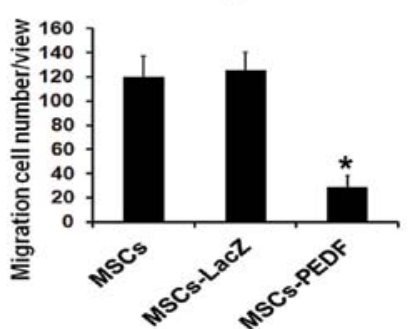

Figure 2. Antiangiogenic activity of PEDF secreted by MSCs-PEDF in vitro. The CM derived from MSCs-PEDF was shown to inhibit tube formation and invasion of human umbilical vein endothelial cells. (A) Tube formation was blocked by PEDF produced by MSCs-PEDF (magnification, x100). Tube formations were quantified by counting the tubes in five randomly selected fields (magnification, $\mathrm{x} 50$; ANOVA; "P<0.01). (B) PEDF produced by MSCs-PEDF inhibited HUVEC invasion in the Transwell assay. The data show that CM derived from MSCs-PEDF dramatically inhibited the invasion properties of endothelial cells (ANOVA; $\left.{ }^{*} \mathrm{P}<0.01\right)$.

A

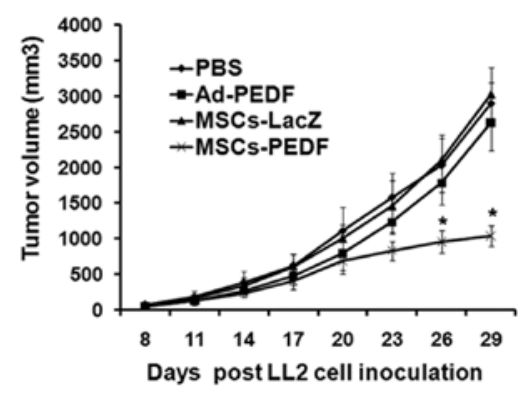

B

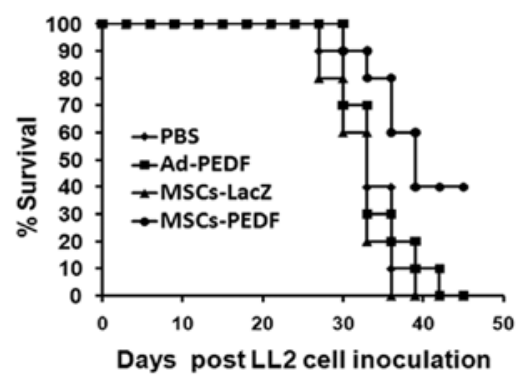

Figure 3. Antitumor effect in mouse Lewis lung carcinoma model. Female C57BL/6 mice received s.c. injection of 5x10 $\mathrm{LLC}$ cells in $100 \mu \mathrm{l}$ of PBS. Treatment with $100 \mu \mathrm{l}$ PBS, $10^{8}$ PFU Ad-PEDF, 5x10 $0^{5}$ MSCs-LacZ, 5x10 MSCs-PEDF were administered i.v. from the tail vein at 4 day intervals for two times. (A) A significant decrease of tumor volumes in PEDF-expressing MSCs-treated mice, compared with the controls (ANOVA; * $\mathrm{P}<0.01$ ), was found in this model. (B) Effects of MSCs-PEDF on the survival of mice inoculated with LLC cells. Analysis of survival was conducted by a log-rank test ("P<0.01).
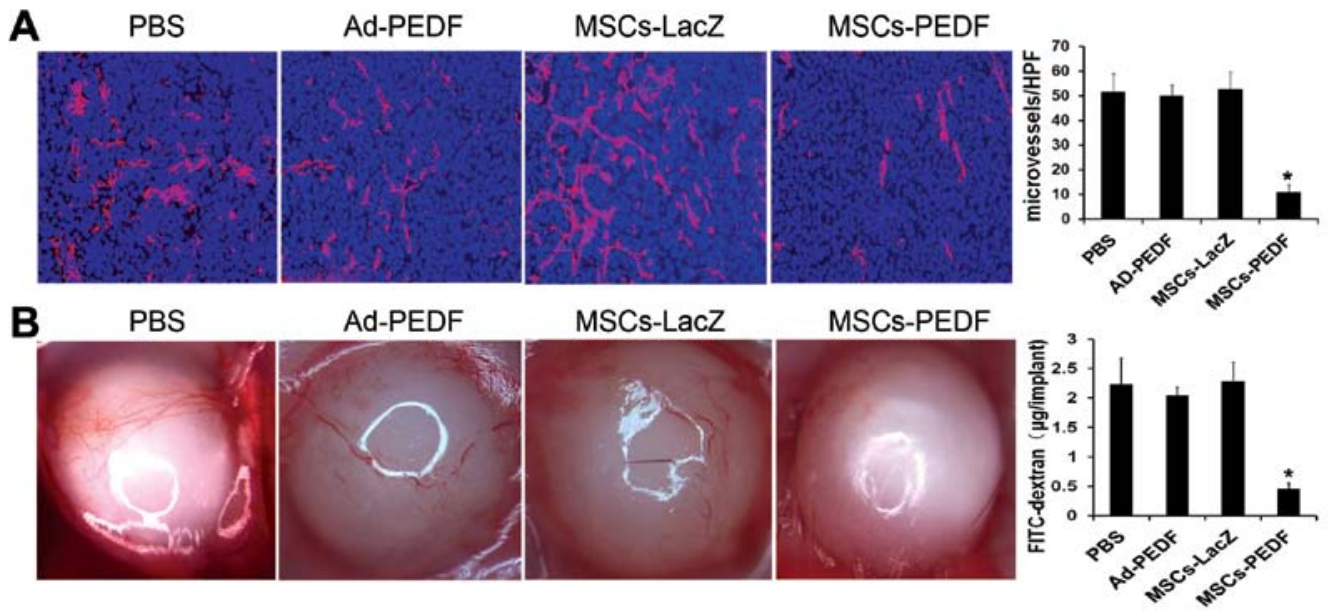

Figure 4. Evaluation of the anti-angiogenetic effect by CD31 immunofluorescence staining and the alginate assay. (A) Sections of frozen LLC tumor tissues obtained from mice treated with PBS, Ad-PEDF, MSCs-LacZ, MSCs-PEDF were stained with anti-CD31 antibody (magnification, x200). Microvessel density $(M V D)$ was determined by counting the number of microvessels per high-power field (magnification, $\mathrm{x} 200$ ) in sections. The MSCs-PEDF group shows a significant decrease of MVD compared to the control groups (ANOVA; " $\mathrm{P}<0.01$ ). (B) Photographs show the surface of alginate beads from different groups. FITC-dextran uptake of alginate implants showed the reduction of vascularization in mice treated with Ad-PEDF compared to mice in the control groups (ANOVA; ${ }^{*} \mathrm{P}<0.01$ ). 


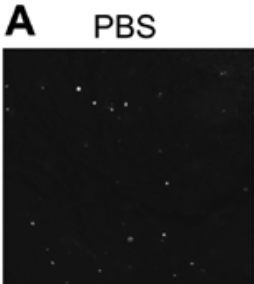

MSCs-LacZ

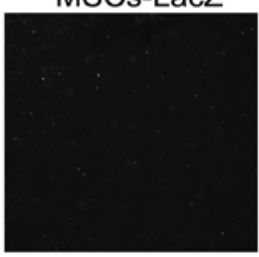

Ad-PEDF

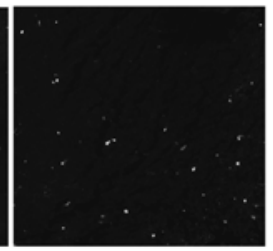

MSCs-PEDF

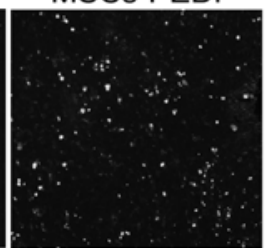

B

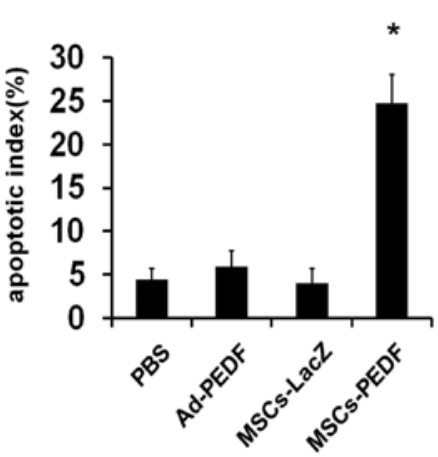

Figure 5. Apoptosis assay by TUNEL staining. (A) Terminal uridine deoxynucleotidyl transferase 2'-deoxyuridine 5-triphosphate nick end labeling assay revealed only a few positive nuclei in tumor tissues of controls including PBS, Ad-PEDF, and MSCs-LacZ groups respectively, but many positive nuclei in the MSCs-PEDF group. (B) Bottom columns represent ultimate apoptotic indexes for tumors from different tumor models. Values are expressed as means \pm SEM. (ANOVA; ${ }^{*} \mathrm{P}<0.01$ ).
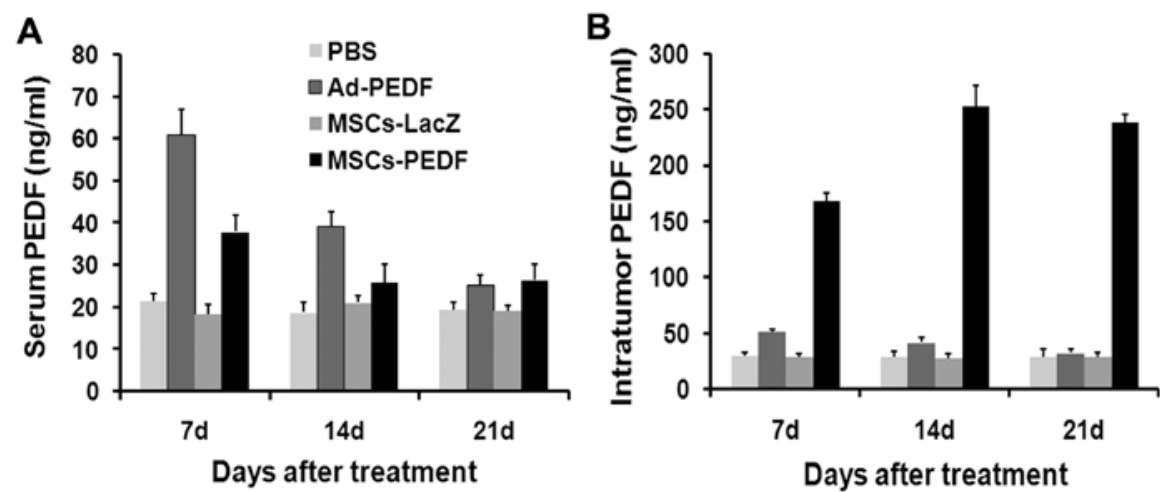

Figure 6. ELISA for expressed PEDF in serum and tumor tissue. The systemic and local concentration of PEDF was detected by dynamic enzyme-linked immunosorbent assay once a week after i.v. administration. In the MSCs-PEDF group, (A) the serum levels of PEDF were $\sim 38 \mathrm{ng} / \mathrm{ml}$ on Day 7 , and declined to $\sim 26 \mathrm{ng} / \mathrm{ml}$ after that; meanwhile, (B) the intratumoral levels of PEDF rose from $\sim 168 \mathrm{ng} / \mathrm{mg}$ ( $7 \mathrm{days})$ to $\sim 253 \mathrm{ng} / \mathrm{mg}$ (14 days), and then slightly declined to $\sim 238 \mathrm{ng} / \mathrm{mg}$ in the 28 days, $\sim 7$-fold vs. the intratumoral level in the free Ad-PEDF group (32 ng/mg). In the free Ad-PEDF group, the levels of PEDF, (A) diminished quickly from $\sim 60$ to $25 \mathrm{ng} / \mathrm{ml}$ in serum and (B) from $\sim 50$ to $\sim 32 \mathrm{ng} / \mathrm{mg}$ intratumorally. Other controls including PBS, MSCs-LacZ hardly led to any elevation of serum or intratumoral levels of PEDF.

intratumorally. Other controls including PBS, MSCs-LacZ hardly led to any elevation of serous or intratumoral levels of PEDF.

\section{Discussion}

In this study, we demonstrated that systemically delivered MSCs loaded with the PEDF gene could preferentially home to tumor loci and induce antiangiogenesis as well as apoptosis of cancer cells, resulting in the inhibition of tumor growth and prolongation of lifespan in the mouse Lewis lung carcinoma (LLC) model.

The choice of PEDF over other cytokines for antitumor effects on LLC in our studies is based on its potent antiangiogenic activity, which has shown to be more effective than any other known endogenous angiogenic inhibitor, including angiostatin, thrombospondin-1, and endostatin. Moreover, PEDF can also exert its antitumor activity via tumor differentiation and direct tumor suppression by apoptosis. It has been reported that PEDF can inhibit several types of tumors including lung carcinomas (8). Purified recombinant PEDF and PEDF-based viral vectors have been investigated in a lot of studies. Nevertheless, a suboptimal half-life in plasma reduces their possible therapeutic effects $(14,25)$. To overcome this shortcoming, a novel and more effective vehicle is needed. In this study, we demonstrated that the engineered MSCs can secret high-level PEDF and the conditioned media derived from these engineered MSCs was capable of inhibiting tube formation and migration of human umbilical vein endothelial cells in vitro.

MSCs are attractive as a cellular vehicle for delivery of antitumoral agents in tumor gene therapy, since they possess several unique biological features. First, the poorer immunogenicity of MSCs enables them to survive for hundreds of days in the host, which can offer a relatively long effect for chronic disease (31). Second, the capacity of MSCs homing to malignant tissues can offer a higher concentration of antineoplastic drugs in the local tumor sites and lower side effect on other healthy organs $(21,31)$. Last, MSCs are easy to harvest, isolate, expand and to gene modify in vitro, which means the offer convenience and cost effectiveness in future preclinical or clinical applications. 
It has been shown that the migratory ability of MSCs was not affected by viral transduction in vitro and in vivo $(21,32,33)$. As a relatively perfect candidate of cellular vehicles used in our study, MSCs could protect PEDF to escape from being destroyed due to entering the circulatory system and being accompanied by potential adverse effects owing to interfering spontaneously with other systems and thereby improving its pharmacokinetics. MSCs were capable of migrating to the tumor sites after intravenous infusion; by contrast, free Ad-PEDF lacks this attribute $(32,33)$. In our study, consecutive administration of free Ad-PEDF could only partly retard the tumor growth to some extent in the midway stage; yet, with potential systemic side effects to the hosts. This also has no therapeutic effect in the ultimate stage. This phenomenon could be partly attributed to the temporary high serum yet low intratumor levels of PEDF as a result of nonselective expression and subsequent elimination of free Ad-PEDF.

Our results suggest that the antitumor activity of MSC-PEDF cells is associated not with the serum concentration of PEDF but rather with the engraftment of MSCs in tumors and the local effects of the PEDF they produce on malignant cells. Therefore, selective engraftment of MSC in tumors is an important issue for further clinical development of this method. Despite the antitumor effects of MSCs-PEDF observed, there was an absence of higher systemic levels of the transgenic protein, suggesting the local effects of MSCs-produced PEDF in the tumor microenvironment. These findings originally suggest that i.v. infused MSCs can circulate over the lung vascular bed and migrate into s.c. growing tumors, supporting the concept that MSCs have the ability of homing into tumor sites. Therefore, therapy with MSCs-PEDF cells could be used to increase PEDF expression in tumors and surrounding tissues and to control the growth of malignant cells. In fact, the potential clinical application of MSCs-PEDF cells could be broad, because many types of malignancies are sensitive to the antiproliferative or proapoptotic effects of PEDF in vitro. The data indicate that systemic administration of MSCs-PEDF led to a pronounced growth inhibition of established subcutaneous LLC and prolonged survival of mice.

In conclusion, our investigation reveals the potential of genetically modified MSC expressing PEDF for Lewis lung cancer. This may prefigure an integrated cellular and molecular therapeutic weapon targeted against advanced human malignancies. However, the same approach could be explored in the delivery of other agents with antitumor activities.

\section{Acknowledgements}

The authors thank members of the State Key Laboratory of Biotherapy for helpful discussions. This study was supported by the National Natural Science Foundation (30973507) and the National 973 Basic Research Program of China (2010CB529900 and 2010CB529906).

\section{References}

1. Ferlay J, Autier P, Boniol M, Heanue M, Colombet M and Boyle P: Estimates of the cancer incidence and mortality in Europe in 2006. Ann Oncol 18: 581-592, 2007.

2. Parkin DM, Bray F, Ferlay J and Pisani P: Global cancer statistics, 2002. CA Cancer J Clin 55: 74-108, 2005.
3. Ettinger DS, Akerley W, Bepler G, et al: Non-small cell lung cancer. J Natl Compr Cancer Netw 8: 740-801, 2010.

4. Jemal A, Siegel R, Ward E, Hao Y, Xu J and Thun MJ: Cancer statistics, 2009. CA Cancer J Clin 59: 225-249, 2009.

5. Folkman J and Shing Y: Angiogenesis. J Biol Chem 267: 10931-10934, 1992.

6. van Hinsbergh VW, Collen A and Koolwijk P: Angiogenesis and anti-angiogenesis: perspectives for the treatment of solid tumors. Ann Oncol 10 (Suppl 4): S60-S63, 1999.

7. Dass CR, Tran TM and Choong PF: Angiogenesis inhibitors and the need for anti-angiogenic therapeutics. J Dent Res 86: 927-936, 2007.

8. Ek ET, Dass CR and Choong PF: Pigment epithelium-derived factor: a multimodal tumor inhibitor. Mol Cancer Ther 5: 1641-1646, 2006.

9. Steele FR, Chader GJ, Johnson LV and Tombran-Tink J: Pigment epithelium-derived factor: neurotrophic activity and identification as a member of the serine protease inhibitor gene family. Proc Natl Acad Sci USA 90: 1526-1530, 1993.

10. Dawson DW, Volpert OV, Gillis P, et al: Pigment epitheliumderived factor: a potent inhibitor of angiogenesis. Science 285: 245-248, 1999.

11. Doll JA, Stellmach VM, Bouck NP, et al: Pigment epitheliumderived factor regulates the vasculature and mass of the prostate and pancreas. Nat Med 9: 774-780, 2003.

12. Hase R, Miyamoto M, Uehara H, et al: Pigment epitheliumderived factor gene therapy inhibits human pancreatic cancer in mice. Clin Cancer Res 11: 8737-8744, 2005.

13. Guan M, Yam HF, Su B, et al: Loss of pigment epithelium derived factor expression in glioma progression. J Clin Pathol 56: 277-282, 2003.

14. Yang J, Chen S, Huang X, et al: Growth suppression of cervical carcinoma by pigment epithelium-derived factor via anti-angiogenesis. Cancer Biol Ther 9: 967-974, 2010.

15. Wang L, Schmitz V, Perez-Mediavilla A, Izal I, Prieto J and Qian C: Suppression of angiogenesis and tumor growth by adenoviralmediated gene transfer of pigment epithelium-derived factor. Mol Ther 8: 72-79, 2003.

16. Roorda BD, ter Elst A, Kamps WA and de Bont ES: Bone marrow-derived cells and tumor growth: contribution of bone marrow-derived cells to tumor micro-environments with special focus on mesenchymal stem cells. Crit Rev Oncol Hematol 69: 187-198, 2009.

17. Pittenger MF, Mackay AM, Beck SC, et al: Multilineage potential of adult human mesenchymal stem cells. Science 284: 143-147, 1999.

18. Eliopoulos N, Al-Khaldi A, Crosato M, Lachapelle K and Galipeau J: A neovascularized organoid derived from retrovirally engineered bone marrow stroma leads to prolonged in vivo systemic delivery of erythropoietin in nonmyeloablated, immunocompetent mice. Gene Ther 10: 478-489, 2003.

19. Seo SH, Kim KS, Park SH, et al: The effects of mesenchymal stem cells injected via different routes on modified IL-12mediated antitumor activity. Gene Ther 18: 488-495, 2011.

20. Galderisi U, Giordano A and Paggi MG: The bad and the good of mesenchymal stem cells in cancer: Boosters of tumor growth and vehicles for targeted delivery of anticancer agents. World J Stem Cells 2: 5-12, 2010.

21. Studeny M, Marini FC, Champlin RE, Zompetta C, Fidler IJ and Andreeff M: Bone marrow-derived mesenchymal stem cells as vehicles for interferon-beta delivery into tumors. Cancer Res 62 : 3603-3608, 2002.

22. Jaffe EA, Nachman RL, Becker CG and Minick CR: Culture of human endothelial cells derived from umbilical veins. Identification by morphologic and immunologic criteria. J Clin Invest 52: 2745-2756, 1973.

23. Eliopoulos N, Francois M, Boivin MN, Martineau D and Galipeau J: Neo-organoid of marrow mesenchymal stromal cells secreting interleukin-12 for breast cancer therapy. Cancer Res 68: 4810-4818, 2008.

24. Bobis S, Jarocha D and Majka M: Mesenchymal stem cells: characteristics and clinical applications. Folia Histochem Cytobiol 44: 215-230, 2006.

25. Yang LP, Cheng P, Peng XC, et al: Antitumor effect of adenovirus-mediated gene transfer of pigment epithelium-derived factor on mouse B16-F10 melanoma. J Exp Clin Cancer Res 28: $75,2009$.

26. Liu JY, Wei YQ, Yang L, et al: Immunotherapy of tumors with vaccine based on quail homologous vascular endothelial growth factor receptor-2. Blood 102: 1815-1823, 2003. 
27. Pang X, Yi Z, Zhang X, et al: Acetyl-11-keto-beta-boswellic acid inhibits prostate tumor growth by suppressing vascular endothelial growth factor receptor 2-mediated angiogenesis. Cancer Res 69: 5893-5900, 2009.

28. Zhang S, Cao Z, Tian H, et al: SKLB1002, a novel potent inhibitor of VEGF receptor 2 signaling, inhibits angiogenesis and tumor growth in vivo. Clin Cancer Res 17: 4439-4450, 2011.

29. Mohamedali KA, Kedar D, Sweeney P, et al: The vascular-targeting fusion toxin VEGF121/rGel inhibits the growth of orthotopic human bladder carcinoma tumors. Neoplasia 7: 912-920, 2005

30. Taraboletti G and Giavazzi R: Modelling approaches for angiogenesis. Eur J Cancer 40: 881-889, 2004.
31. Fritz V and Jorgensen C: Mesenchymal stem cells: an emerging tool for cancer targeting and therapy. Curr Stem Cell Res Ther 3 32-42, 2008

32. Chen X, Lin X, Zhao J, et al: A tumor-selective biotherapy with prolonged impact on established metastases based on cytokine gene-engineered MSCs. Mol Ther 16: 749-756, 2008.

33. Gao Y, Yao A, Zhang W, et al: Human mesenchymal stem cells overexpressing pigment epithelium-derived factor inhibit hepatocellular carcinoma in nude mice. Oncogene 29: 2784-2794, 2010. 